\title{
Transurethral flexible ureteroscope incision and drainage combined with Holmium laser for the treatment of parapelvic renal cysts: A Retrospective Study
}

\author{
Xiangjun Meng ( $\square$ mengzij@sina.com ) \\ Dongguan People's Hospital \\ Qiwu Mi \\ Dongguan People's Hospital \\ Derong Chen \\ Dongguan People's Hospital
}

\section{Research Article}

Keywords: Renal cysts, Flexible ureteroscopy, Holmium laser, Incision, Drainage

Posted Date: January 31st, 2022

DOI: https://doi.org/10.21203/rs.3.rs-1272966/v1

License: (c) (i) This work is licensed under a Creative Commons Attribution 4.0 International License.

Read Full License 


\section{Abstract}

Background-We aimed to investigate the clinical efficacy and safety of transurethral flexible ureteroscope incision and drainage combined with Holmium laser for the treatment of parapelvic renal cysts.

Methods-The clinical data of 65 patients with parapelvic renal cysts were retrospectively analyzed between October 2017 and April 2021. Among them, 31 patients with parapelvic cysts (group 1) underwent a transurethral flexible ureteroscope incision and drainage combined with Holmium laser, and the other 34 patients (group 2) underwent retroperitoneal laparoscopic unroofing. The clinical characteristics of patients were recorded. The surgery time, intraoperative blood loss, hospitalization time, complications and cyst size at 1 year postoperatively were recorded and statistically analyzed.

Results-All patients were successfully treated with flexible ureteroscope incision and drainage or retroperitoneal laparoscopic unroofing. No statistically significant difference was found between group 1 and 2 in terms of clinical characteristics, including age, gender, body mass index (BMI), location, cyst size, and Bosniak classification of renal cysts. Group 1 demonstrated shorter surgery time, less intraoperative blood loss, and shorter hospitalization time than the control group (group 2) $(p<0.001)$. However $\$ no significant differences in complications and cyst size at 1 year postoperatively was observed between the two groups $(p>0.05)$.

Conclusions-The transurethral flexible ureteroscope incision and drainage with Holmium laser for the treatment of parapelvic renal cysts has obvious advantages over traditional surgery, and is worthy of promotion and application, but its long-term effect needs further follow-up.

\section{Introduction}

Renal cyst is a common urinary system. Studies have found that the incidence of renal cyst is $50 \%$ of adults older than 50 years, and $66 \%$ of adults will have formed renal cysts by the age of 80 years [1]. Parapelvic renal cyst is a special type of renal cystic disease [2], and a non-hereditary disease, especially a cyst adjacent to renal pelvis. Kiryluk and Gupta [3] named renal cysts originating from renal sinus and around the renal pelvis or renal sinus as parapelvic cysts.

Clinical symptoms of parapelvic cysts are usually atypical. Parapelvic cyst can be diagnosed by ultrasonography, enhanced computed tomography (CT), magnetic resonance imaging and other imaging examinations. Parapelvic cysts are usually benign lesions with slow progression. If the cyst size is small, no self-conscious symptoms or complications are observed, and imaging examination shows no obvious renal pelvic compressions, conservative observation and regular follow-up should be conducted. Due to its special position, parapelvic cyst easily pressure on renal vessels or pelvis, and cause hypertension, hydronephrosis and other symptoms. Therefore, active treatment should be initiated when symptoms of oppression occur. 
Laparoscopic renal cyst unroofing is the preferred treatment option for parapelvic cyst [4]. With the development of minimally invasive technology, Yu et al [5] reported that the incision and drainage under ureteroscope was performed to treat parapelvic cysts. However, rare study compared the procedure to laparoscopic renal cyst unroofing. Therefore, this study aimed to report the clinical efficacy and safety of transurethral flexible ureteroscope incision and drainage, comparing with retroperitoneal laparoscopic unroofing for the treatment of parapelvic renal cysts.

\section{Materials And Methods}

The Ethics Committee of Dongguan People's Hospital (Dongguan, China) approved the study. All individual participants agreed to publish informed consent forms with detailed information, and provided signed informed consent.

Between October 2017 and April 2021, 65 with parapelvic renal cysts treated by the transurethral flexible ureteroscope incision and drainage or retroperitoneal laparoscopic unroofing participated in this study. Among them, 31 patients with parapelvic cysts (group 1) underwent a transurethral flexible ureteroscope incision and drainage combined with Holmium laser, and the other 34 (group 2) underwent retroperitoneal laparoscopic unroofing. Surgeon with 10-year experience performed surgeries according to standard procedures. Patients' clinical characteristics are reported in Table 1. Preoperative intravenous urography, ultrasonography and CT were performed to diagnose parapelvic cysts. Retrograde pyelography was performed as necessary. Patients with parapelvic cysts of $>3 \mathrm{~cm}$ were included in this study. Patients with parapelvic cysts suspected as malignant according to CT were excluded. In addition, those with uncontrolled urinary tract infection, urethral or ureteral stricture, hemorrhagic diseases and cardiopulmonary insufficiency were excluded.

Urine analysis, urine culture, and serum biochemical tests were performed in all patients preoperatively. Patients with infection cannot undergo the procedures until the infection was controlled. All patients were administered a prophylactic dose of prophylactic antibiotics 30 min preoperatively. All patients were followed up 1, 3, and 12 months postoperatively in outpatient department. The follow-up examination included ultrasonography and CT.

\section{Surgical protocol.}

A total of 31 patients (group 1) underwent a transurethral flexible ureteroscope incision and drainage combined with Holmium laser. A ureteral double $\mathrm{J}$ tube was routinely indwelled to dilate ureter 2 weeks preoperatively. The procedure was performed under general anesthesia in lithotomy position. Initially, a rigid ureteroscope (F8.0/9.8 Wolf) was retrogradely inserted into renal pelvis to explore the ureter, and ureteral access sheath (Flexor 12/14F, Cook) was placed along guide wire. Then, flexible ureteroscope (URF-V, OLYMPUS) was inserted into the renal pelvis through the ureteral access sheath, and the incision and drainage of the parapelvic cysts were performed with a Holmium laser device (LUMENIS Versa Pulse Power Suite), the frequency of Holmium laser of $30 \mathrm{~Hz}$ and energy of $0.8 \mathrm{~J}$ (Fig. 1). If it was difficulty for surgeon to identify cyst, methylene blue was injected into the cyst, the cyst wall became blue, which could 
help surgeon identify the cyst wall accurately (Fig. 2). Finally, a ureteral stenting (JJ stent) was routinely indwelled with upper end inside the cyst for 4 weeks.

In group 2, 34 accepted the retroperitoneal laparoscopic unroofing. All of them were treated by the retroperitoneal approach. The procedure was performed under general anesthesia in the standard left/right lateral decubitus position. First, three working ports $(0.5,0.5$, and $1.0 \mathrm{~cm}$, respectively) were placed. Then, kidneys were isolated, especially the area adjacent to the position of the cyst. The cyst was unroofed $0.5 \mathrm{~cm}$ adjacent to the renal parenchyma. The cystic wall was sent for pathologic analysis. A tube (22 French) used for drainage was placed in the retroperitoneum.

Statistical analysis.

Data were presented as mean \pm standard deviation (SD) or number. Age, BMI, cyst size, surgery time, blood loss, hospitalization time and cyst size at 1 year postoperatively were in normal distribution. Student's t-test was used to compare continuous variables between groups, and the chi-square test was used to compare categorical variables. SPSS 17.0 (SPSS, Chicago, IL, USA) was used for statistical analysis. Significance was established at $\mathrm{P}<0.05$.

\section{Results}

A total of 65 patients were successfully treated with transurethral flexible ureteroscope incision and drainage or retroperitoneal laparoscopic unroofing. Among them, 31 with parapelvic cyst (group 1) underwent a transurethral flexible ureteroscope incision and drainage combined with Holmium laser, and the remaining 34 (group 2) underwent the retroperitoneal laparoscopic unroofing. In group 2, one patient failed in transurethral flexible ureteroscope incision and drainage because surgeon could not identify the cyst wall, and thus retroperitoneal laparoscopic unroofing was performed instead. Comparison of clinical and perioperative factors between the transurethral flexible ureteroscope incision and retroperitoneal laparoscopic unroofing is shown in Table 2.

The mean age was $47.6 \pm 8.7$ and $46.8 \pm 7.8$ years in groups 1 and 2 , respectively, without significant difference between the two groups $(p=0.701)$. No significant difference in gender was observed between group 1 and group $2(p=0.73)$. No significant difference was found between two groups in terms of BMI ( $23.8 \pm 2.2$ vs. $\left.24.1 \pm 2.0 \mathrm{~kg} / \mathrm{m}^{2}, p=0.551\right)$. No significant difference with regard to the location of renal cyst was observed between the two groups $(p=0.271)$. Ultrasonography and CT were preoperatively performed to measure the size of renal cyst. The size of the renal cyst was $5.3 \pm 0.9 \mathrm{~min}$ in group 1 and $5.1 \pm 0.9 \mathrm{~min}$ in group 1 , and there was no significant difference between the two groups $(p=0.333)$. According to CT diagnosis, there were 31 patients with Bosniak category I renal cyst in group 1, 33 Bosniak category II renal cyst and 1 Bosniak category II renal cyst in group 2, without significant difference between group 1 and group $2(p=0.336)$.

The surgery time in group 1 was shorter than that in group 2, and the difference between two groups was statistically significant ( $30.1 \pm 4.3$ vs. $54.4 \pm 6.4 \mathrm{~min}, \mathrm{p}<0.001$ ). Blood loss was $5.5 \pm 1.7 \mathrm{ml}$ and $59 \pm 9.9$ 
$\mathrm{ml}$ in groups 1 and 2 , respectively, with significant difference between the two groups $(\mathrm{p}<0.001)$. The hospitalization time in group 1 was shorter than that in group 2, with statistically significant difference between the two groups ( $4.5 \pm 0.8$ vs. $5.6 \pm 0.9$ days, $p<0.001)$. The follow-up examination was postoperatively performed to measure the size of the renal cyst. No significant difference was observed between the two groups in terms of cyst size at 1 year postoperatively $(1.0 \pm 0.9 \mathrm{vs} .0 .6 \pm 0.6 \mathrm{~cm}, p=$ 0.106). No severe complications were observed in the two groups. In group 1 , significant hemorrhage was noted in 1 patient, which lasted for 2 days postoperatively. One patient had transient fever $\left(38.7^{\circ} \mathrm{C}\right.$ temperature) in group 2, but no significant difference was found between the two groups $(p=0.947)$.

\section{Discussion}

Renal cyst is a common disease in urology, and its incidence increases with age. Studies have found that the incidence of renal cyst is approximately $50 \%$ in adults older than 50 years, and $66 \%$ of adults will have formed renal cysts aged 80 years [1]. Parapelvic renal cyst is a special type of renal cystic disease [2], and is non-hereditary, especially a cyst adjacent to renal pelvis. Kiryluk [3] named renal cysts originating from renal sinus and around the renal pelvis or renal sinus as parapelvic cysts. Kutcher $\mathrm{R}$ [6] believed that chronic inflammation led to the local expansion of pelvic lymphatic vessels and caused parapelvic cysts.

As the parapelvic cysts grow slowly, they are usually asymptomatic. Parapelvic cysts cause symptoms by compressing renal collecting system and renal vessels. The common symptoms include lumbar pain, hypertension, hematuria, repeated urinary tract infection, and urinary tract obstruction $[7,8]$. Parapelvic cysts need surgical intervention when cysts with relatively larger size cause symptoms.

To date, various methods have been used for the treatment of renal cysts, including percutaneous sclerotherapy, unroofing by open surgery, laparoscopic unroofing, and drainage procedure by ureteroscope. Compared with simple renal cysts, the treatment of parapelvic cyst is relatively difficult due to the cyst location adjacent to renal pelvis and vessels $[9,10]$. Percutaneous sclerotherapy is simple and economical; however, its recurrence rate is high due to the existence of cyst wall. In addition, because the parapelvic cyst is adjacent to the renal hilum and pelvis, sclerotherapy could cause severe pyelonephritis or secondary ureteropelvic junction obstruction $[11,12,13]$. In the past, laparoscopic unroofing was the preferred treatment for parapelvic cyst. However, the laparoscopic unroofing is difficult for most surgeons. Because of the deep position of parapelvic cyst, renal pelvis and vessels were easily injured intraoperatively $[14,15]$. The study reported that the incidence of pelvic injury was $9.5 \%$ during the laparoscopic unroofing [8].

With the development of minimally invasive technology, Basiri et al $[15,16,17]$ reported that ureteroscope incision and internal drainage was applied in the treatment of parapelvic cyst. In 1991, Kavoussi et al [18] reported they successfully performed the ureteroscope incision and internal drainage by ureteroscopy. They considered that this method has advantages of minimally invasive, less postoperative pain, and rapid recovery. In this study, 31 patients successfully underwent a transurethral flexible ureteroscope 
incision and drainage combined with Holmium laser. Under the flexible ureteroscope, the visual field was not limited, and pelvis and all calyces were observed. The flexible ureteroscope can reach the target calyces, and incise parapelvic cyst. Compared with the method of retroperitoneal laparoscopic unroofing, no significant difference was observed in terms of cyst size at 1 year postoperatively.

The key of ureteroscope incision and drainage is to find and identify renal cyst under flexible ureteroscope. In order to avoid renal parenchyma or renal vessel injury, the incision should be located in the thin wall of parapelvic cyst. The typical wall of parapelvic cyst looks transparent. However, the surface of some parapelvic cysts is the same as that of the renal pelvis; therefore, it is difficult to identify the parapelvic cysts under the flexible ureteroscope [19]. When methylene blue is injected into the cyst, the cyst wall becomes blue, which can help surgeons accurately identify the cyst wall [20]. For parapelvic cysts in the posterior part of kidney, percutaneous renal puncture was performed under B-ultrasound, the puncture needle inserted into the renal pelvis through the cyst, and then incision was performed along the puncture. In this study, only one patient failed to undergo transurethral flexible ureteroscope incision and drainage because the surgeon could not identify the cyst wall.

Some limitations exist in this study. First, due to its retrospective nature, selection bias may occur in our study. Second, because this is a single center study, the number of patients is rather small, and further prospective randomized research is needed. Third, the transurethral flexible ureteroscope incision and drainage have several disadvantages, such as higher costs and two hospitalizations.

\section{Conclusions}

In summary, the transurethral flexible ureteroscope incision and drainage with Holmium laser for the treatment of parapelvic renal cysts has obvious advantages over the traditional surgery, and is worthy of the promotion and application, but its long-term effect needs further follow-up studies.

\section{Abbreviations}

CT : enhanced computed tomography

SD : standard deviation

BMI: indicates Body Mass Index

\section{Declarations}

\section{Ethical approval and consent to participate}

The Ethics Committee of Dongguan People's Hospital (Dongguan, China) approved the study, which adhered to the International Conference on Harmonisation Good Clinical Practice standards. Ethics approval no.: XJS2018-009. In this study, all patients signed a preoperative informed consent form and 
agreed to participate in the accompanying scientifc research and all methods were performed in accordance with the Declaration of Helsinki.

\section{Consent for publication}

Written informed consent was obtained from the patient for publication of the case.

\section{Availability of data and materials}

The data used to support the findings of this study are available from the corresponding author upon request.

\section{Conpeting interests}

No competing interests exist.

\section{Acknowledgements}

We thank the medical staff (Department of Urology, Dongguan People's Hospital) for supporting the study.

\section{Funding}

The authors have not received external funding for this study.

\section{Author ' contribution}

Xiangjun Meng: coordination, design of the study, data collection, interpretation of data, writing the article, tables, final revision.

Derong Chen: writing, data collection, figures, tables, final revision.

Qiwu Mi: design of the study, statistical analysis,interpretation of data.

\section{Author details}

1. Department of Urology, Dongguan people's Hospital, Dongguan, Guangdong, P.R. China.

No.78, Wandao Road, Wanjiang Street, Dongguan City, Guangdong, P.R. China.

${ }^{*}$ Corresponding author】Xiangjun Meng, E-mail address: mengzij@sina.com

0086-0769-28636983(phone), 0086-0769-22222353 (fax), E-mail address: mengzij@sina.com.

Qiwu Mi

Department of Urology, Dongguan people's Hospital, Dongguan, Guangdong, P.R. China. 
No.78, Wandao Road, Wanjiang Street, Dongguan City, Guangdong, P.R. China.

0086-0769-28636986 (phone), 0086-0769-22222353 (fax), 1440428102@qq.com.

Derong Chen

Department of Urology, Dongguan people's Hospital, Dongguan, Guangdong, P.R. China.

No.78, Wandao Road, Wanjiang Street, Dongguan City, Guangdong, P.R. China.

E-mail address: menglinhui96@126.com.

\section{References}

1. Laucks SP Jr, McLachlan MS. Aging and simple cysts of the kidney. Br J Radiol 1981; 54: 12-14.

2. P. Ao, L. Shu, D. Zhuo, Z.X. Zhang, C.B. Dong, H.B. Huang, Z.Q. Wei. Risk factors associated with systemic inflammatory response syndrome after flexible ueteroscopic lithotripsy based on enhanced recovery after surgery. Zhonghua Yi Xue Za Zhi 2019; 99: 758-763. doi: 10.3760/cma.j.issn.03762491.2019.10.010.

3. Kiryluk K, Gupta M. A large obstructive parapelvic cyst: challenging diagnosis and management. Kidney International 2019; 71: 955. doi: 10.1038/sj.ki.5002124.

4. Ozcan L, Polat EC, Onen E, Cebeci OO, Memik O, Voyvoda B, Ulukaradag E, Kara BY. Comparison between retroperitoneal and transperitoneal approaches in the laparoscopic treatment of bosniak typelrenal cysts: A retrospective study. Urol J. 2019; 12: 2218-2222.

5. Yu W, Zhang D, He X, Zhang Y, Lia G, Deng G, Jin B. Flexible ureteroscopic management of symptomatic renal cystic diseases. J Surg Res. 2015; 196: 118-123.

6. Kutcher R, Mahadevia P, Nussbaum MK, Rosenblatt R, Freed S. Renal peripelvic multicystic lymphangiectasia. Urology 1987; 30:177-179.

7. Umemoto Y, Okamura T, Akita H, Yasui T, Kohri K. Clinical evaluation of parapelvic renal cysts: do these represent latent urological malignant disease? Asian Pac J Cancer Prev. 2019; 10: 1119-1120.

8. Camargo AH, Cooperberg MR, Ershoff BD, Rubenstein JN, Meng MV, Stoller ML. Laparoscopic management of peripelvic renal cysts: University of California, San Francisco, experience and review of literature. Urology 2005; 65: 882-887.

9. Desai D, Modi S, Pavicic M, Thompson M, Pisko J. Percutaneous renal cyst ablation and review of the current literature. Journal of Endourology Case Reports 2016: 2: 11-13. doi:

10.1089/cren.2015.0013.

10. Mao X, Xu G, Wu H, Xiao J. Ureteroscopic management of asymptomatic and symptomatic simple parapelvic renal cysts. BMC Urol. 2015; 6: 15:48.

11. Hoenig DM, McDougall EM, Shalhav AL, Elbahnasy AM, Clayman RV. Laparoscopic ablation of peripelvic renal cysts. J Urol. 1997; 158: 1345-1348. 
12. Akinci D, Gumus B, Ozkan OS, Ozmen MN, Akhan O. Single-session percutaneous ethanol sclerotherapy in simple renal cysts in children: long-term follow-up. Pediatr Radiol. 2005; 35:155158.

13. Perdzyński W, Klewar M, Rutka J, Stembrowicz Z, Sakson B. Simple renal cysts in children: treatment with ethyl alcohol injection into their lumen. Pol Merkur Lekarski 2000; 8: 46-248.

14. Nasseh H, Hamidi Madani A, Ghanbari A, Arfa S. Laparoscopic unroofing of symptomatic kidney cysts. A single center experience. Minerva Urol Nefrol. 2013; 6: 285-289.

15. Basiri A, Hosseini SR, Tousi VN, Sichani MM. Ureteroscopic management of symptomatic, simple parapelvic renal cyst. J Endourol. 2010; 24: 537-540.

16. Mancini V, Cormio L, d'Altilia N, Benedetto G, Ferrarese P, Balzarro M, Defidio L, Carrieri G. Retrograde Intrarenal Surgery for Symptomatic Renal Sinus Cysts: Long-Term Results and Literature Review. Urol Int. 2018; 101: 150-155. doi: 10.1159/000488685.

17. Junwen Shen, Yu Chen, Rongjiang Wang. Efficacy and Complication of Flexible Ureteroscopic Holmium Laser Incision for Simple Renal Cysts: A Retrospective Study. J Endourol. 2019; 33: 881886. doi: 10.1089/end.2019.0515.

18. Kavoussi LR, Clayman RV, Mikkelsen DJ, Meretyk S. Ureteronephroscopic marsupialization of obstructing peripelvic renal cyst. J Urol. 1991; 146: 411-414.

19. Bas O, Nalbant I, Can Sener N, Firat H, Yeşil S, Zengin K, Yalcınkaya F, Imamoglu A. Management of renal cysts. JSLS. 2015; 19: e2014 00097. doi: 10.4293/JSLS.2014.00097.

20. Zhixian Wang, Xiaoyong Zeng, Chong Chen, Tao Wang, Ruibao Chen, Jihong Liu. Methylene Blue Injection via Percutaneous Renal Cyst Puncture Used in Flexible Ureteroscope for Treatment of Parapelvic Cysts: A Modified Method for Easily Locating Cystic Wall. Urology 2019; 125:243-247. doi: 10.1016/j.urology.2018.11.014.

\section{Tables}

Table 1 Clinical characteristics of the study population. 


\begin{tabular}{|ll|}
\hline Variables & Mean ( $(\mathrm{SD})$ or $\mathrm{N}(\mathrm{\Xi})$ \\
\hline Patients $(\mathrm{n})$ & 65 \\
\hline Age (years) & $48.5 \pm 6.2$ \\
\hline${ }^{*}$ BMI $\left(\mathrm{kg} / \mathrm{m}^{2}\right)$ & $23.2 \pm 4$ \\
\hline Location(n) & $34(52.3)$ \\
Right & $31(47.7)$ \\
Left & $64(98.5)$ \\
\hline Bosniak classification & $1(1.5)$ \\
Bosniak I & $4.8 \pm 0.3$ \\
Bosniak II & \\
*Cyst size (cm) & $30(46.2)$ \\
\hline Gender (n) & $35(53.8)$ \\
\hline Male & \\
\hline
\end{tabular}

${ }^{\square} S D$ indicates Standard Deviation;

*BMI indicates Body Mass Index;

*Cyst size $=$ the diameter of stone based on preoperative CT scanning.

Table 2 Comparison of clinical and perioperative factors between flexible ureteroscope incision (group 1) and retroperitoneal laparoscopic unroofing (group 2). 


\begin{tabular}{|llll|}
\hline Variable & Group 1 & Group 2 & P-value \\
\hline Patients $(\mathrm{n})$ & 31 & 34 & NA \\
\hline Age (years) & $47.6 \pm 8.7$ & $46.8 \pm 7.8$ & 0.701 \\
\hline Gender (n) & & & 0.73 \\
Male & $15(48.4)$ & $15(51.6)$ & \\
Female & $16(44.1)$ & $19(55.9)$ & \\
\hline${ }^{*}$ BMI (kg/m $\left.{ }^{2}\right)$ & $23.8 \pm 2.2$ & $24.1 \pm 2.0$ & 0.551 \\
\hline Location & & & 0.271 \\
Right & $14(46.2)$ & $20(58.8)$ & \\
Left & $17(54.8)$ & $14(41.2)$ & \\
\hline *Cyst size (cm) & $5.3 \pm 0.9$ & $5.1 \pm 0.9$ & 0.333 \\
\hline Bosniak classification of renal cysts & & & 0.336 \\
\hline Bosniak I & $31(100)$ & $33(97.1)$ & \\
Bosniak II & $0(0)$ & $1(2.9)$ & \\
\hline Surgery time (min) & $30.1 \pm 4.3$ & $54.4 \pm 6.4$ & $<0.001$ \\
\hline Blood loss (ml) & $5.5 \pm 1.7$ & $59 \pm 9.9$ & $<0.001$ \\
\hline Hospitalization time (days) & $4.5 \pm 0.8$ & $5.6 \pm 0.9$ & $<0.001$ \\
\hline Cyst size at 1 year postoperatively (cm) & $1.0 \pm 0.9$ & $0.6 \pm 0.6$ & 0.106 \\
\hline Complications (n) & 1 & 1 & 0.947 \\
\hline
\end{tabular}

Data are presented as mean \pm SD or number (percent);

पNA indicates not applicable;

${ }^{*}$ BMI indicates Body Mass Index;

*Cyst size $=$ the diameter of stone based on preoperative CT scanning .

\section{Figures}



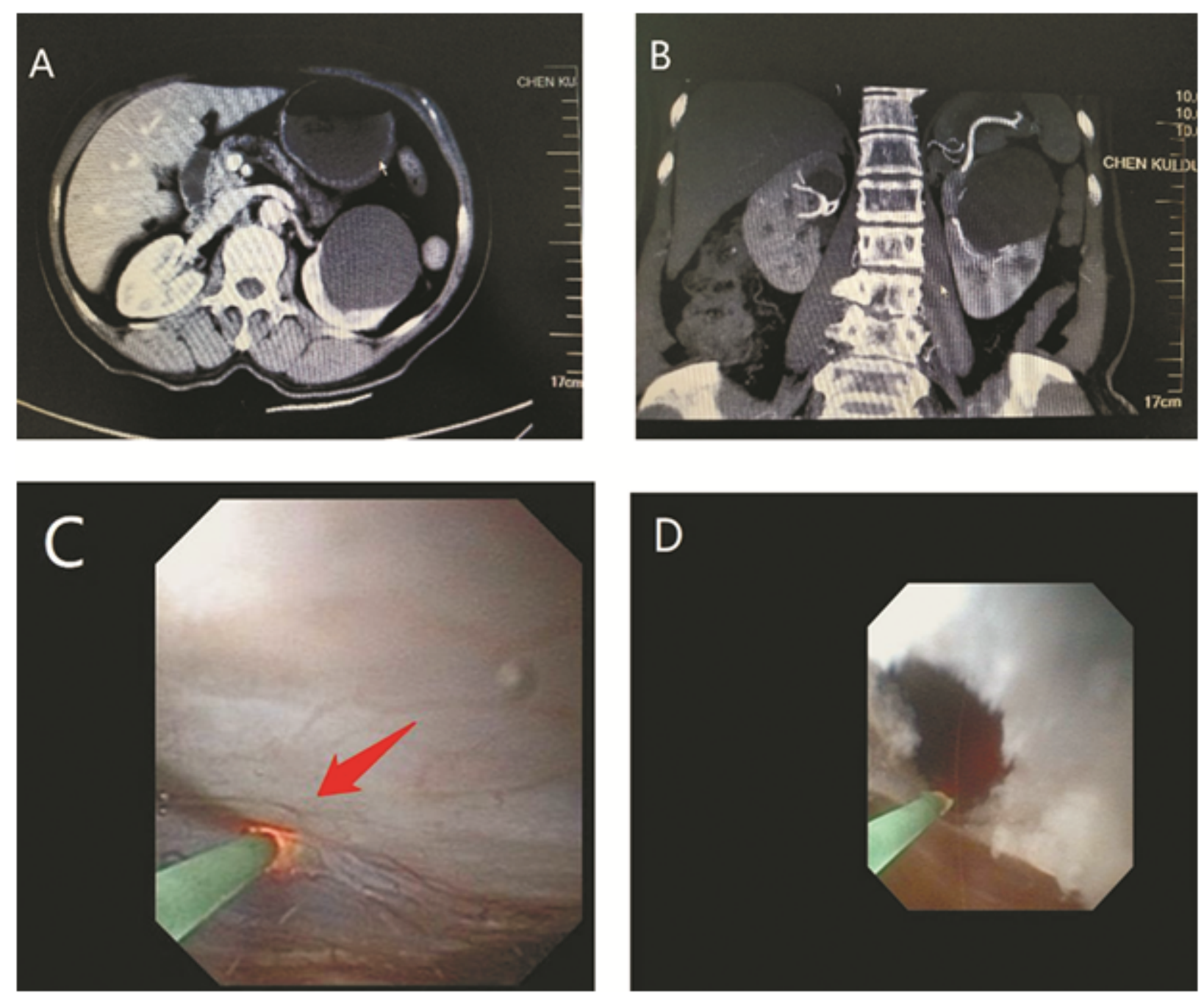

\section{Figure 1}

A 67-year-old woman underwent a transurethral flexible ureteroscope incision and drainage. The maximum intensity projection image showed parapelvic cyst in the left kidney ( $A$ and $B$ ). The typical wall (red arrow) of the parapelvic cyst looked transparent (C). The image of the parapelvic renal cyst after the flexible ureteroscope incision and drainage (D). 

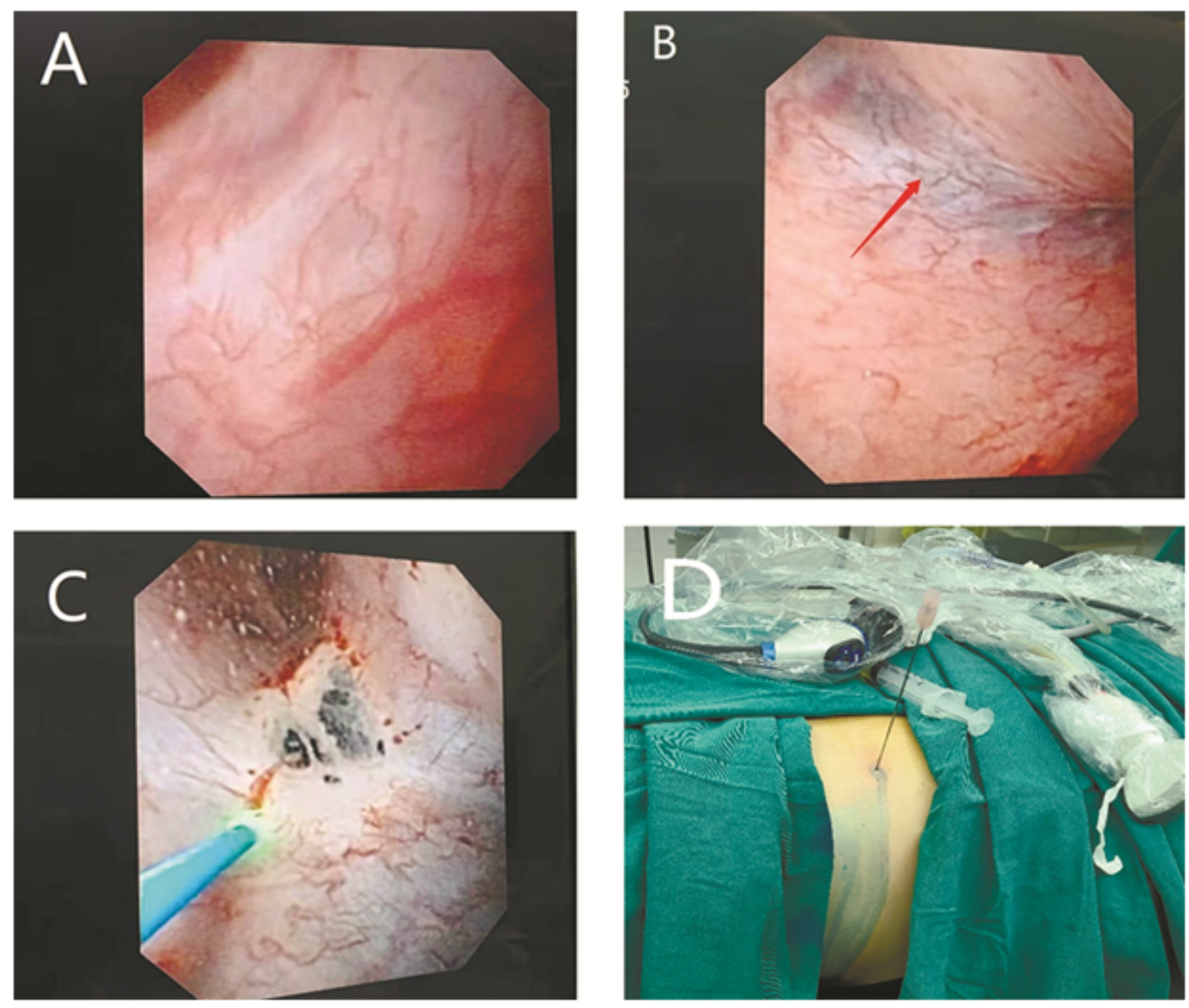

\section{Figure 2}

The left parapelvic cyst was identified by injecting methylene blue. The cyst wall before the injection of methylene blue is shown(A). The methylene blue was injected into the cyst to identify the parapelvic cyst, and the cyst wall (red arrow) became blue (B). The parapelvic cyst was incised by holmium laser (C). Percutaneous renal puncture (D). 